\title{
3D Models Coding and Morphing for Efficient Video Compression
}

\author{
Franck Galpin $^{1}$, Raphaèle Balter ${ }^{2,3}$, Luce Morin ${ }^{2}$ and Koichiro Deguchi ${ }^{1}$ * \\ ${ }^{1}$ Graduate School of \\ Information Sciences \\ Tohoku University, Sendai \\ Japan \\ ${ }^{3}$ France-Telecom R\&D \\ rue du Clos Courtel \\ Cesson-Sévigné \\ France
}

\begin{abstract}
This paper presents new results in the field of video coding and compression using $3 D$ information. Contrary to prior art in $3 D$ model-based coding where $3 D$ models have to be known, the $3 D$ models are here automatically computed from the original video sequence. The camera parameters and the scene content are supposed unknown and we only assume a static scene viewed by a moving monocular camera. The video sequence is then processed on the fly. A stream of $3 D$ models is extracted and compressed, using adapted computer vision and compression techniques. The visualization of the reconstructed video sequence uses an adapted morphing method. We finally show results obtained with the proposed compression scheme, compared with the H26L compression standard. We demonstrate the efficiency of our approach, especially in the field of very low bitrate coding.
\end{abstract}

\section{Introduction}

Video compression is based on the modeling of image contents and suppression of redundant information among the images of an input video sequence. On the coder side, a set of successive images (called a GOP for Group of Pictures) is replaced by a more compact parameterized model and some residual information compensating model inadequacies. On the decoder side, the transmitted model parameters and residual information are used to reconstruct the original set of images. For instance, in the standard video compression algorithms MPEG1-2 and H26x, the video sequence is modeled as a grid of square blocks of pixels moving with translational motion. In MPEG4 coding scheme, it is possible to replace the square grid by a set of arbitrary shaped patches. Such schemes allow to compress video sequences of arbitrary size and contents. On the other side, 3D

\footnotetext{
*email: \{galpin,kodeg\}efractal.is.tohoku.ac.jp, \{raphaele.balter, luce.morin\}eirisa.fr
}

model based coding aims at representing the video sequence with one or severals textured 3D models and a set of camera/objects parameters. This method provides very efficient compression and it has been studied for many years, particularly in the field of video-conference where a 3D model of the human face is used to represent the video sequence of the speaker [1]. It also provides 3D manipulation functionalities, such as illumination changes, virtual object insertion, stereoscopic visualization or the generation of virtual viewpoints. However, it requires that the scene content is known and that an a priori known 3D model is available both at the coder and the decoder [2] [3]. In this paper, we propose to apply the principle of 3D model-based coding for scenes with unknown static contents viewed by a moving monocular camera.

As an a priori known 3D model is unavailable, it is automatically extracted from the input video sequence using shape from motion methods developed in computer vision [4] [5] [6]. In the case of video compression, the aim of 3D reconstruction is not to recover an exact geometric model of the scene but to obtain a compact description of the video sequence. The 3D model is thus an intermediate representation between the original encoded video and the visualized decoded video. As for "impostors" in computer graphics, it may not be a faithful 3D model of the actual scene, as long as it enables to reconstruct the original images.

Based on this idea, we propose to estimate a set of local $3 \mathrm{D}$ models, instead of a unique one containing all the information viewed in the entire video sequence, as in previous automatic shape-from-motion schemes [7] [8]. This choice has several advantages :

- Global consistency of extracted 3D information is not required. This allows to simplify estimation and use inaccurate camera parameters.

- Global illumination changes along time are modeled and reconstructed.

- Sequences of arbitrary size can be processed with on the fly estimation and streaming of the 3D models. 
- Camera motion is unconstrained as long as it is not degenerated (for example a pure rotation).

Our first experiments have validated this scheme [9]. However, the reconstructed sequence shows visual artifacts, low objective quality and abrupt changes at the end of GOPs. We here present a geometry and texture morphing process which overcomes these effects and ensures a smooth transition between 3D models. This method can be related to view-dependent texture mapping [10]. We also reduce geometric distortions on the model shape by proposing a depth adapted quantization law.

In the following, we first briefly describe the extraction of the 3D models and then pay more attention on the coding and decoding schemes. Results on real video sequences are finally shown and discussed.

\section{3D models stream generation}

We first present the structure of the representation: each 3D model is extracted and used for a small portion of the video sequence called a GOP (Group Of Picture). The GOPs overlap themselves, that is the last image of a GOP is the same as the first image of the next one (see fig. 1). We call these images keyframes. Keyframes are automatically selected adaptively to video contents, based on several criteria depending on motion, percentage of outgoing points in images and $3 \mathrm{D}$ reconstruction stability [11].

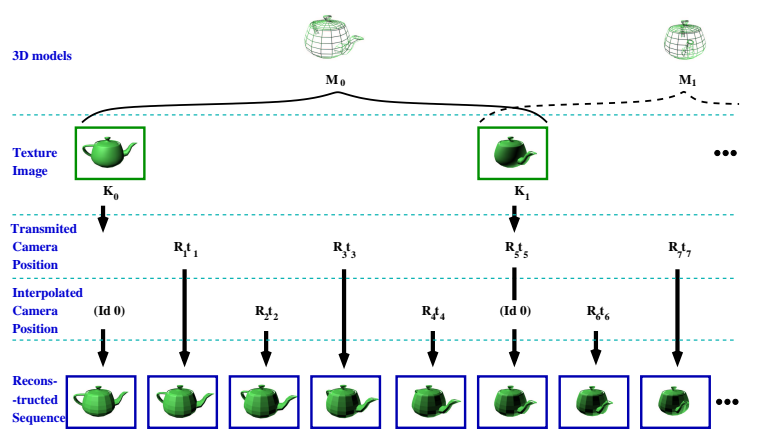

Figure 1: Reconstruction of the original sequence

For each GOP, a 3D model is automatically extracted on the fly. The principle of extraction is based on shape-frommotion method: we use a dense mesh-based motion estimator using multi-grid and multi-resolution approaches [12].

The camera intrinsic parameters are estimated using a self-calibration algorithm or fixed to approximate values. Accurate intrinsic parameters are not required since we design our representation to be robust to inaccurate intrinsic parameters. The extrinsic parameters are computed using classical calibration method and an adapted bundle adjustment method [11]. The dense motion field from the first to the last image of the GOP and camera parameters for these two images allow to reconstruct a dense depth map of the first image of the GOP. Figure 2 shows an example of such a depth map extracted from the Street video sequence (see results section).
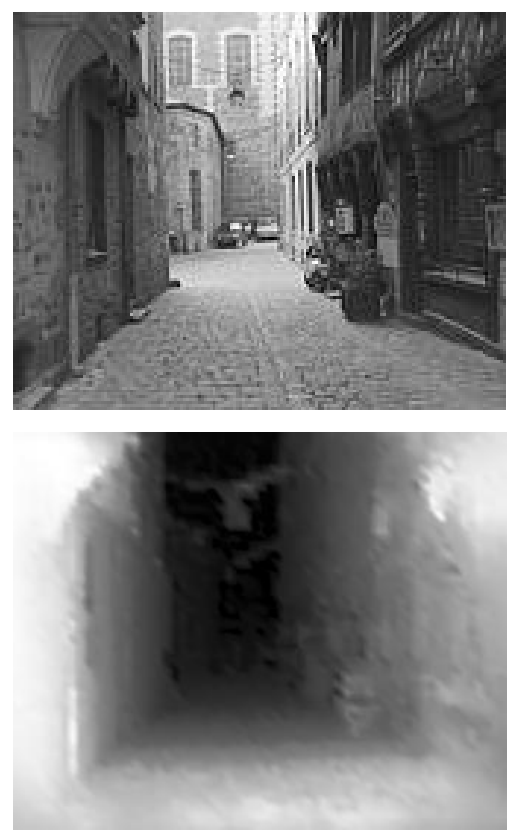

Figure 2: An example of a depth map (bottom) extracted from the Street video sequence. The top figure is the corresponding image in the sequence.

A uniform triangular mesh is then applied on each first image of each GOP which gives a 3D model and its associated texture image (the first image of the GOP). Camera extrinsic parameters are then retrieved for each image in the video sequence using a pose estimation algorithm.

\section{Stream compression}

Once the original video sequence has been encoded as a stream of 3D models, it is compressed using adapted compression techniques for the different parts of the representation (3D model, texture images and camera parameters).

\subsection{Texture images}

An IPP scheme is used, as in MPEG for low bitrate, that is: one Intra (I) image at the beginning of the video stream followed by Predicted (P) images. Intra images do not depend 
of other images, whereas Predicted images depend on the previously decoded image.

The first image of the sequence $T_{0}$ (Intra type) is compressed using a method similar to JPEG2000 standard [13], that is wavelet transform, adaptive quantization (using $E$ $B C O T)$ and entropic coding.

The following texture images (Predicted type) are predicted using the previously decoded texture image (of the previous GOP) $T_{n}$ and the previous 3D model $M_{n}$ :

$$
\tilde{T}_{n+1}=\operatorname{Pr}\left(M_{n}, T_{n}, C_{n+1}\right)
$$

where $C_{n+1}$ is the camera associated with keyframe $T_{n+1}$ and $\operatorname{Pr}(M, T, C)$ denotes the perspective projection of model $M$ textured with image $T$ onto camera $C$.

It produces a prediction image of the texture image $\tilde{T}_{n+1}$ similar to a forward motion compensated image in classical video coding scheme (see fig. 3). We then encode the difference image $E_{n+1}$ using the same method as for the Intra image.

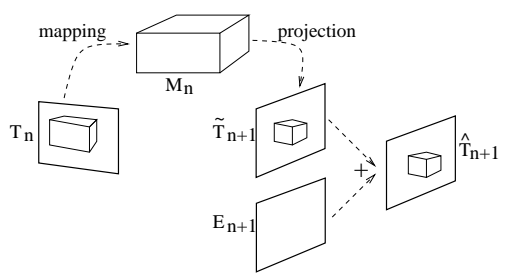

Figure 3: Principle of predictive coding method of the texture images. The predicted texture image $\tilde{T}_{n+1}$ is computed by transfer of the previous keyframe $T_{n}$ using the previous 3D model $M_{n}$.

\subsection{D models}

3D models can be seen as being extruded from the $\mathrm{u}-$ niformly meshed depth maps. Each 3D model, that is the decimated depth map, is compressed using wavelet transform and an adapted quantization law: the depth of each vertex of the 3D model is quantized using a normalized inverse law. We show below that it provides a linear dependence between 2D reprojection error and quantization step.

Equations of back-projection for a 3D point $M=$ $(x, y, z)$ are given by:

$$
\begin{aligned}
\tilde{m}_{n} & =K_{n}\left(I_{3} \mid 0_{3}\right) \tilde{M} \\
\tilde{m}_{n+1} & =K_{n+1}(R \mid t) \tilde{M}
\end{aligned}
$$

where $K_{n}$ is the matrix of camera intrinsic parameters at the time of keyframe $n, m_{n}$ denotes the pixel coordinates of the point in the image at this time, and $\tilde{m}_{n}$ denotes the $m_{n}$ vector in homogeneous coordinates. The motion of a pixel is then given by:

$$
d(m)=\left\|m_{n}-m_{n+1}\right\|
$$

We assume that:

- intrinsic parameters $K$ are fixed for a given GOP,

- pixel motion induced by the camera rotation $R$ is insignificant compared to the one induced by the camera translation $t$. This is a valid assumption for the type of video we deal with, such as walks through cities or paths.

With $t=\left(t_{x}, t_{y}, t_{z}\right)$, we then obtain:

$$
m=\left(\begin{array}{c}
\frac{x}{z} \\
\frac{y}{z}
\end{array}\right) \quad \text { and } \quad m_{n+1}=\left(\begin{array}{c}
\frac{x+t_{x}}{z+t_{z}} \\
\frac{y+t_{y}}{z+t_{z}}
\end{array}\right)
$$

That is, for a pure translation:

$$
d^{2}(m) \simeq \frac{\left(x . t_{z}-z \cdot t_{x}\right)^{2}+\left(y \cdot t_{z}-z \cdot t_{y}\right)^{2}}{z^{2} \cdot\left(z+t_{z}\right)^{2}}
$$

The depth $\alpha$ of a 3D point $M$ is defined as its distance to the optical center $O$ of the first camera of the GOP: $M=$ $O+\alpha \vec{u}$ where $\vec{u}$ is the view-line unit vector. From equation (4) we express 2D motion $d$ as a function of depth $\alpha$ and for $\alpha . u_{z}>>t_{z}$, which is a reasonable hypothesis for the scenes we deal with, it comes:

$$
d^{2}(\alpha) \simeq \frac{1}{\alpha^{2}} \cdot \frac{\left(u_{x} \cdot t_{z}-u_{z} \cdot t_{x}\right)^{2}+\left(u_{y} \cdot t_{z}-u_{z} \cdot t_{y}\right)^{2}}{u_{z}^{4}}
$$

that is $d(\alpha)$ is proportional to $1 / \alpha$. It simply implies that, for a fixed depth error, the more a point is far from the optical center, the less the projection error is. We then need to quantify the $\alpha$ values so that the reprojection error which is the error on $d(\alpha)$ is linear with respect to quantization step. In order to obtain values in a target range, we first normalize $\alpha \in\left[0, \alpha_{\infty}\right]$ values using $n(\alpha)=\frac{\alpha}{\alpha_{\infty}} \in[0,1]$, where $\alpha_{\infty}$ is the maximum observed depth for a given scene. We then propose to apply a uniform quantization law on $f(\alpha)$ as

$$
f(\alpha)=V_{\max } \cdot\left(2-\frac{2}{1+n(\alpha)}\right)
$$

This definition allows, for $n(\alpha) \in[0,1]$, to get $f(\alpha) \in$ $\left[0, V_{\max }\right]$. The constant $V_{\max }$ depends on the number of bits used to encode the values, as we encode only integer values. Practically, we use $V_{\max }=2^{16}-1$ (that is we use 16 bits to encode a depth value).

Tests on real data confirm that this quantization law allows to compress the 3D models with a linear reprojection error with respect to the quantization step (see fig. 4). The quantization step is chosen in such a way that the projection 
error is less than 0.5 pixel. The mesh step size also affects the bitrate of the compressed 3D model. We typically fix the mesh step size to 8 for low bitrate and 12 for very low bitrate.

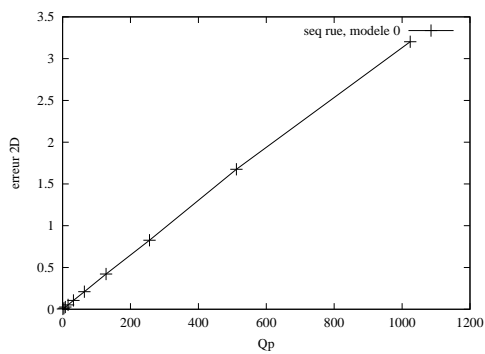

Figure 4: Average 2D reprojection error related to quantization step Qp, for a 3D model extracted from the Street sequence; the reprojection error is computed for each vertex of the 3D model as the distance between projection before and after quantization; it is measured in the last image of the GOP, where it is the largest.

\subsection{Bitrate allocation and adaptation}

The representation is effectively compressed, allowing to compute the real coding cost. Figure 5 shows a typical result on the first 3D model extracted from a real video sequence (see figures 15 and 16). It displays 2D reprojection error for the 3D model, depending on the coding cost per triangle in bits. It shows that the 3D model cost is between 2 and 3 bits per triangle for a reprojection error less than 0.5 pixel, which is very low compared to the texture cost. This is confirmed with figure 6: the texture image cost remains the major coding cost in the proposed representation.

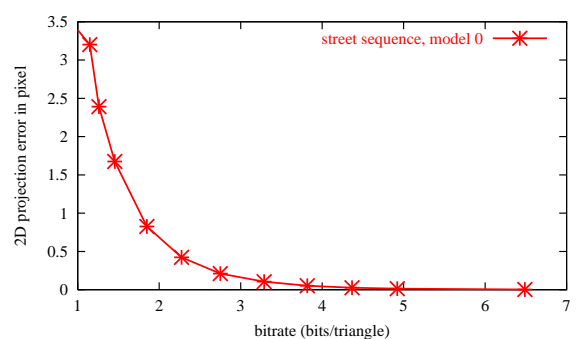

Figure 5: Typical bitrate obtained with the adapted quantization.

The target bitrate $R_{c}$ can thus be arbitrarily fixed thanks to $E B C O T$ coding of texture images. The coding cost $R_{t e x t}$ of the current texture image is simply chosen as:

$$
R_{t e x t}=\frac{R_{c} \cdot\left(t_{n+1}-t_{n}\right)}{f r}-\left(R_{c a m}+R_{M}\right)
$$

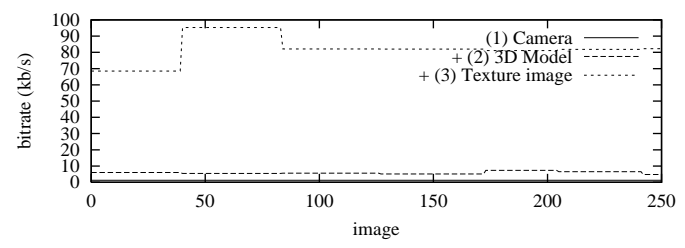

Figure 6: Coding cost of each part of the representation: (1) camera cost, (2) adding the 3D model cost, (3) adding the texture image cost.

with $f r$ the framerate of the video sequence, $R_{c a m}$ the coding cost of the camera parameters, $R_{M}$ the coding cost of the $3 \mathrm{D}$ model and $t_{n}$ the time at GOP $n$.

\section{Video sequence Reconstruction}

On the decoder side, the original video sequence has to be reconstructed based on transmitted information.

\subsection{Principle}

All images inside a GOP are reconstructed by projecting the associated textured 3D model on each camera viewpoint in the GOP, as illustrated in figure 1. More precisely, for reconstructing current image $I_{c}$ in GOP $n$, the 3D model $M_{n}$ textured with keyframe image $T_{n}$ is projected onto current viewpoint $C_{c}$ :

$$
\hat{I}_{c}=\operatorname{Pr}\left(M_{n}, T_{n}, C_{c}\right)
$$

where $\operatorname{Pr}(M, T, C)$ denotes the perspective projection of model $M$ textured with image $T$ onto camera $C$. As the 3D model is a $3 \mathrm{D}$ triangular mesh associated with texture coordinates in texture image $T_{n}$, classical rendering techniques and dedicated hardware can be used for real time decoding.

However, because this method uses only the texture of the first image in the GOP, the reconstructed image quality decreases along the GOP, and a visual abrupt change occurs at the transition between two successive 3D models.

To ensure a smooth transition between GOPs, we propose an adapted texture fading and geometric morphing procedure.

\subsection{Texture fading}

The texture image used for the current GOP is not $T_{n}$ but a linear combination of the current model texture $T_{n}$ and the next model texture $T_{n+1}$ ( figure 7).

Thus, current image $I_{c}$ is reconstructed as

$$
\begin{aligned}
\hat{I}_{c} & =\operatorname{Pr}\left(M_{n}, T_{c}, C_{c}\right) \\
\text { with texture image } T_{c} & =(1-\gamma) T_{n}+\gamma \tilde{T}_{n+1}
\end{aligned}
$$




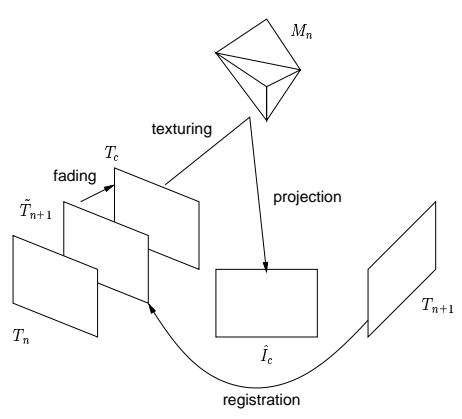

Figure 7: Texture fading: $T_{n+1}$ is registered and combined with $T_{n}$ to produce faded texture image $T_{c}$. 3D model $M_{n}$ is textured with $T_{c}$ and projected to reconstruct image $\hat{I}_{c}$.

The fading factor $\gamma$ reflects the current camera position, relatively to the total translation from first to last image in the GOP :

$$
\gamma=\frac{\left\|t_{c}-t_{t_{n}}\right\|}{\left\|t_{t_{n+1}}-t_{t_{n}}\right\|}
$$

where $t_{t}$ denotes camera translation vector at time $t$. To ensure a correct superposition of textures $T_{n}$ and $T_{n+1}$ during summation, texture image $T_{n+1}$ is first registered into $\tilde{T}_{n+1}=\operatorname{Pr}\left(M_{n}, T_{n+1}, C_{n}\right)$, which is $3 \mathrm{D}$ model $M_{n}$ textured with $T_{n+1}$ and viewed from camera $C_{n}$.

\subsection{Geometric morphing}

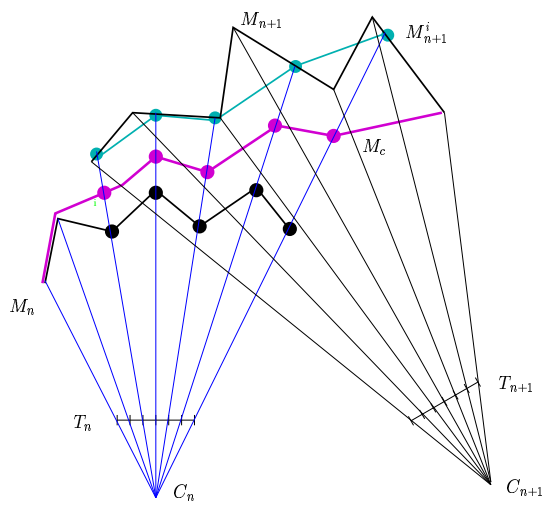

Figure 8: Geometric morphing: $M_{n+1}$ is remeshed into $M_{n+1}^{i}$. Intermediate model $M_{c}$ is then interpolated between $M_{n}$ and $M_{n+1}^{i}$ along camera $C_{n}$ view-lines.

Similarly, a current 3D model $M_{c}$ is computed for each current viewpoint $C_{c}$ by linear interpolation between models $M_{n}$ and $M_{n+1}$. As there is no vertex to vertex correspondence between $M_{n}$ and $M_{n+1}, M_{n+1}$ is first remeshed into $M_{n+1}^{i}$ so that each vertex in $M_{n}$ has a correspondent in $M_{n+1}^{i}$. This is achieved by intersecting viewlines from camera $C_{n}$ supported by $M_{n}$ vertexes with model
$M_{n+1}$ (see figure 8). Linear interpolation between $M_{n}$ and $M_{n+1}^{i}$ along view lines then provides intermediate shapes, i.e. current model $M_{c}$ as :

$$
M_{c}=(1-\gamma) M_{n}+\gamma M_{n+1}^{i}
$$

with $\gamma$ previously defined.

Texture fading and geometric morphing ensure a smooth transition between GOPs : in particular they take into account global illumination changes along the sequence and reduce texture distortion in the reconstructed sequence (see figures 9-10). They improve not only visual quality but also objective quality: the $P S N R^{1}$ of the reconstructed sequence is higher (see figure 11) and when it would previously decrease constantly along the GOP, it may even increase at the end of GOPs (see PSNR curves presented in the Results section). The drawback is a higher computing cost at the decoder and the necessary pre-fetching of GOP $n+1$ for reconstructing GOP $n$.
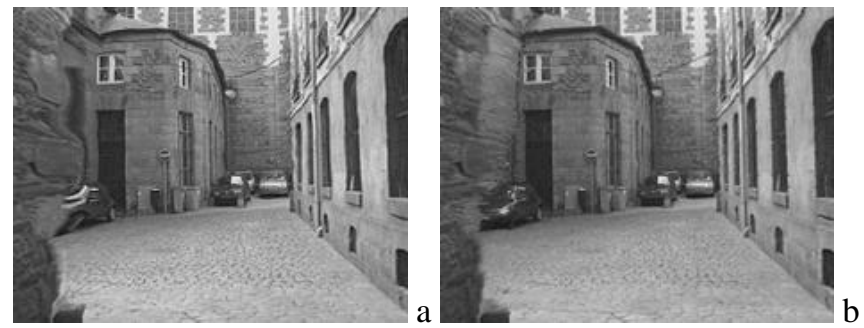

Figure 9: Effect of texture fading on Street sequence (image 240) (a) without fading (b) with texture fading.
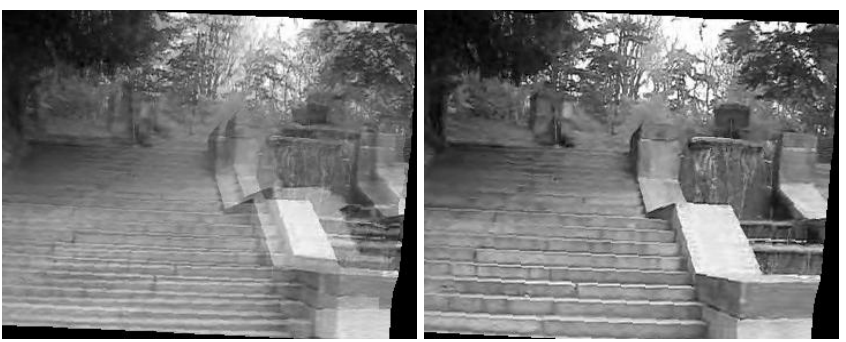

Figure 10: Effect of geometric morphing on Stairway sequence: to visualise the geometric shift, two successive models are projected and superimposed for a viewpoint outside the camera path, without geometric morphing (left) and with geometric morphing(right).

${ }^{1}$ PSNR (Peak Signal-to-Noise Ratio) is defined as $10 \log _{10} \frac{255^{2}}{M S E}$, where $M S E$ is the Mean Square Error between original and reconstructed images. 


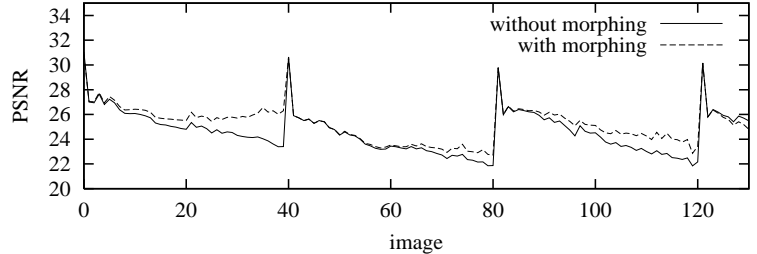

Figure 11: PSNR score on Street video sequence, with and without the fading implementation.

\section{Results}

The compression scheme performance is first compared to the state of the art standard coder (H264/MPEG4-AVC ${ }^{2}$ ) and we show new results in very low bitrate coding area.

Results obtained on the Street video sequence ${ }^{3}$ are first presented: format is CIF 4:2:0 at $25 \mathrm{~Hz}$, global motion is a translation along $\mathrm{z}$-axis, neither camera parameters nor scene content are known. The internal camera parameter$\mathrm{s}$ are fixed to typical values and focal length is fixed to an approximate value. The video sequence is compressed with $\mathrm{H} 26 \mathrm{~L}$ video encoder at $82 \mathrm{~kb} / \mathrm{s}$ which is the minimum bitrate this coder can achieve on this sequence. The sequence is also encoded with the proposed method denoted Rec3D with the same parameters.

Figure (19) shows PSNR scores along the sequence. The Image and $H 26 L$ curves denote the PSNR of the reconstructed sequence with the $\operatorname{Rec} 3 D$ method and the H26L encoder respectively. $P S N R$ scores are globally similar for both methods, and better for the Rec3D method when the $3 \mathrm{D}$ model is refresh (peaks on the second curve). The presented fading and morphing techniques provide quality continuity : PSNR increases progressively at the end of GOPs, thanks to using the next GOP's 3D model and texture which provide a this time a better reconstruction than the current GOP's model and texture. An abrupt quality change is thus avoided when changing GOPs.

Distortion on reconstructed images as shown by the Image curve is produced both by texture distortion (texture image compression artifacts) and geometric distortion (3D model estimation errors and depth compression artifacts). The Texture curve shows the PSNR of the decoded texture image, i.e. the quality obtained with texture distortion alone, without geometric distortion. It is dramatically much better than the final obtained quality shown by the Image curve. The low PSNR value of reconstructed images is thus essentially due to geometric distortion.

On figures (15-18), we compare visual quality of the reconstructed video sequence: the images clearly show that

\footnotetext{
${ }^{2}$ used version is H26L-TML coder v.6. which ovecomes previous MPEG and ITU standards [14]

${ }^{3}$ video sequence from Thomson Multimedia and FTRD
}

Rec $3 D$ provides better visual quality. For such a bitrate, H26L coding introduces classical block artifacts and texture degradation whereas Rec $3 D$ still provides high quality texture images resulting in very good visual quality of the reconstructed video sequence. Note that the better visual quality is not reflected by PSNR curves presented above. This is due to geometric distortion which greatly decreases $P S N R$ when it may have little impact on visual quality (think of a geometric distortion equal to one pixel translation for a demonstrative example). Indeed visual quality of reconstructed images with Rec $3 D$ is better reflected by PSNR on texture images (Texture curve) than PSNR on reconstructed images (Image curve).

Figures (12-14) show similar results on the Stairway video sequence. The format of this sequence is CIF 4:2:0 at $25 \mathrm{~Hz}$, global motion is a translation along $\mathrm{x}$-axis, neither camera parameters nor scene content are known. The internal camera parameters are fixed to typical values and focal length is fixed to an approximate value. One must notice that this video sequence is very shaky. The video sequence is compressed both with the proposed coder and with $\mathrm{H} 26 \mathrm{~L}$ video encoder at $125 \mathrm{~kb} / \mathrm{s}$. This is the minimum possible bitrate reachable by $\mathrm{H} 26 \mathrm{~L}$ for this sequence. Figure 14 shows that the PSNR of H26L coder is better than the one of Rec $3 D$ coder. However, the PSNR of the texture images (Texture curve) and figures (12-13) show that the visual quality of the reconstructed sequence is still better with the $\operatorname{Rec} 3 D$ coder.

Moreover, the proposed method allows coding at very low bitrates (up to $16 \mathrm{~kb} / \mathrm{s}$ for CIF, $25 \mathrm{~Hz}$ format) which are not reachable by classical image based coders. Figures (1721) show results on the Street video sequence for a target bitrate of $16 \mathrm{~kb} / \mathrm{s}$. Figure 21 shows the PSNR score along the video sequence: Texture curve shows that visual quality remains good for such a bitrate. Figures 17 and 20 show an image extracted from the reconstructed video sequence: despite compression artifacts, global visual quality is still good. One must notice that such a bitrate is considered as extremely low for $\mathrm{CIF} / 25 \mathrm{~Hz}$ format video sequence.

\section{Conclusion}

We have proposed a vision computer based approach for video compression. An adapted quantization of depth information together with geometric and texture morphing during visualization allow to significantly improve the visual and objective quality of the reconstructed video sequences. The results on real video sequences demonstrate that this approach is very efficient for low bitrate coding and also enables very low bitrate coding not reachable by curren$\mathrm{t}$ coders. We show that the proposed scheme allows better performance than classical schemes like H26L in the case of static scene video sequences. One must also notice 
that the proposed representation intrinsically allows 3D manipulations like stereo-visualization or scene manipulation [11]. In future work, we plan to extend the proposed scheme to scenes with moving objects, making the approach more general for video coding purpose.

\section{References}

[1] F. Prêteux and M. Malciu, "Model-based head tracking and 3d pose estimation," in Visual Conference on Image Processing, San Jose, California, 1998, pp. 94-110.

[2] B. Girod and al., "3d image models and compression - synthetic hybrid or natural fit?," in Proc. ICIP, Oct. 1999.

[3] G. Martinez, "Joint position estimation for object-based analysis-synthesis coding," in Proc. VCIP, June 2000.

[4] M. Pollefeys, R. Koch, M. Vergauwen, B. Deknuydt, and L. Van Gool, "Three-dimensional scene reconstruction from images," in Proc. SPIE Electronic Imaging, ThreeDimensional Image Capture and Applications III, 2000.

[5] R. Kochand M. Pollefeys and L. Van Gool, "Realistic 3d scene modeling from uncalibrated image sequences," in Proc. ICIP, Oct. 1999.

[6] M. Magnor and B. Girod, "Model-based coding of multiviewpoint imagery," in Proc. VCIP, June 2000.

[7] A. Fitzgibbon and A. Zisserman, "Automatic camera recovery for closed or open image sequences," in Proc. ECCV (1), 1998, pp. 311-326.

[8] D. Nistèr, "Reconstruction from uncalibrated sequences with a hierarchy of trifocal tensors," in Proc. ECCV, Dublin, Ireland, 2000, pp. 649-663.

[9] F. Galpin, L. Morin and K .Deguchi, "Compression performance of computer vision based coding," in Proc. MVA, Nara, Japan, 2002

[10] P. Debevec, C .Taylor and J .Malik, "Modeling and Rendering Architecture from Photographs: A Hybrid Geometryand Image-Based Approach", in SIGGRAPH, Computer Graphics Proceedings, New Orleans, Aug. 1996, pp. 11-20,

[11] F. Galpin and L. Morin, "Sliding adjustment for 3D video representation," in Eurasip Journal on Applied Signal Processing, vol.10, Oct. 2002.

[12] S. Pateux, G. Marquant, and D. Chavira-Martinez, "Objec$\mathrm{t}$ mosaicking via meshes and crack-lines technique. Application to low bit-rate video coding", in Proc. PCS, Seoul, Korea, Apr. 2001, pp. 417-420,

[13] Information Technology, "Jpeg2000 image coding system, iso/iec fdis 15444-1," 2000.

[14] H. Schwarz and T. Wiegrand, "The emerging JVT/H.26L video coding standard", in Proc. IBC, Amsterdam, 2002.

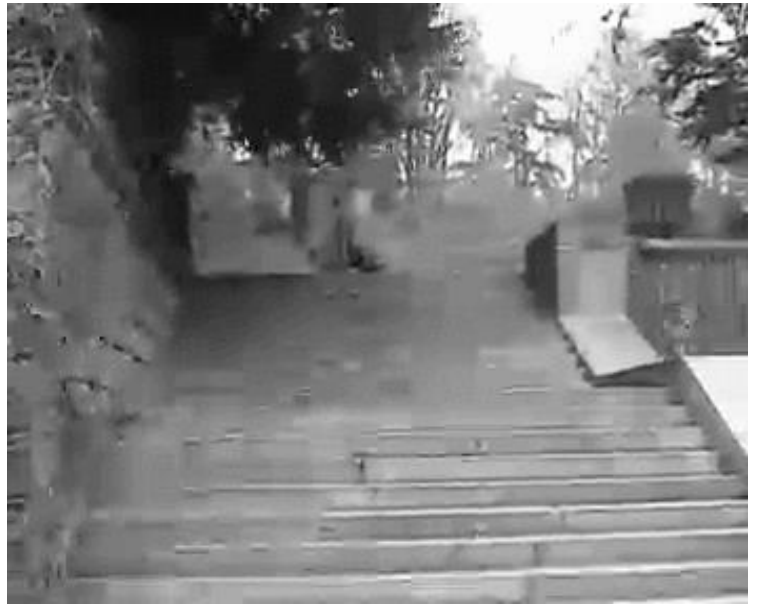

Figure 12: Stairway video sequence at $125 \mathrm{~kb} / \mathrm{s}$ : image 67 reconstructed by $\mathrm{H} 26 \mathrm{~L}$ coder.

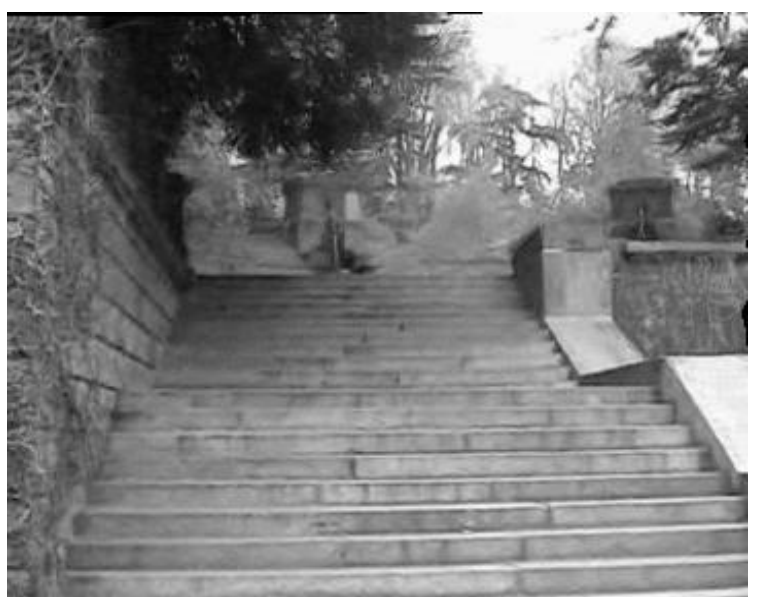

Figure 13: Stairway video sequence at 125kb/s: image 67 reconstructed by Rec3D coder.

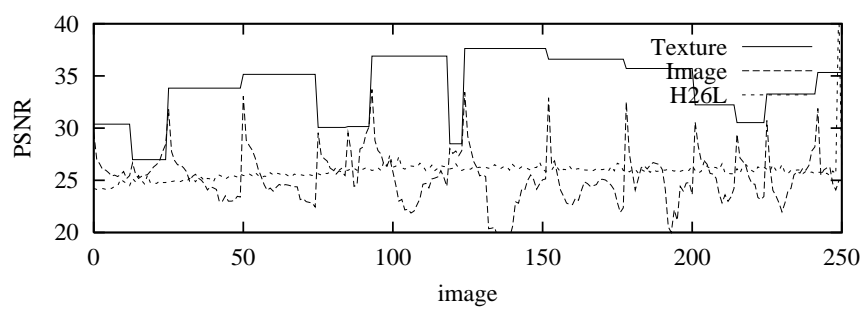

Figure 14: PSNR of Stairway video sequence at $125 \mathrm{~kb} / \mathrm{s}$ : comparison between H26L and Rec3d method. 


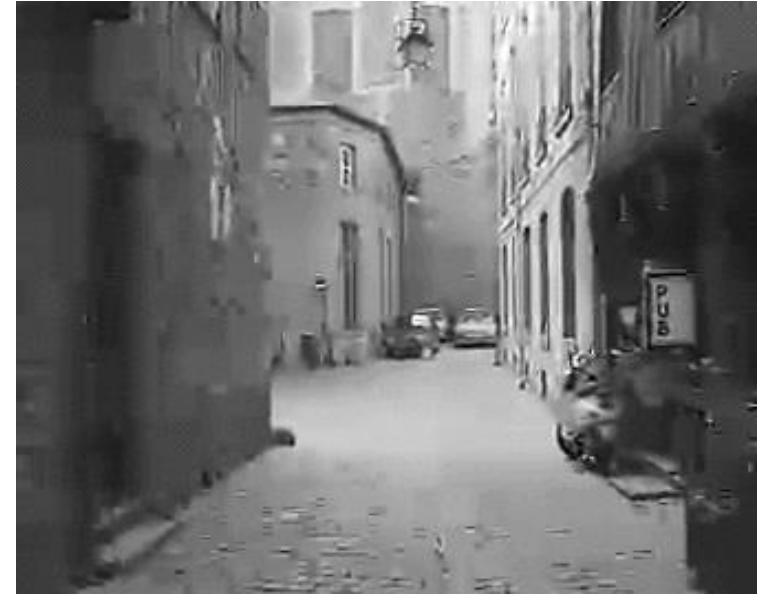

Figure 15: Street video sequence at $82 \mathrm{~kb} / \mathrm{s}$ : image $100 \mathrm{re-}$ constructed by H26L coder.

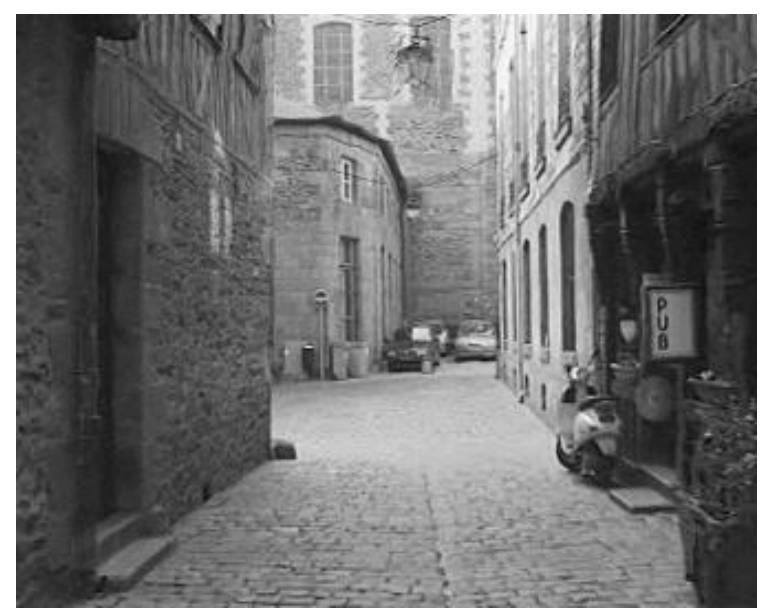

Figure 16: Street video sequence at $82 \mathrm{~kb} / \mathrm{s}$ : image 100 reconstructed by Rec3D coder.

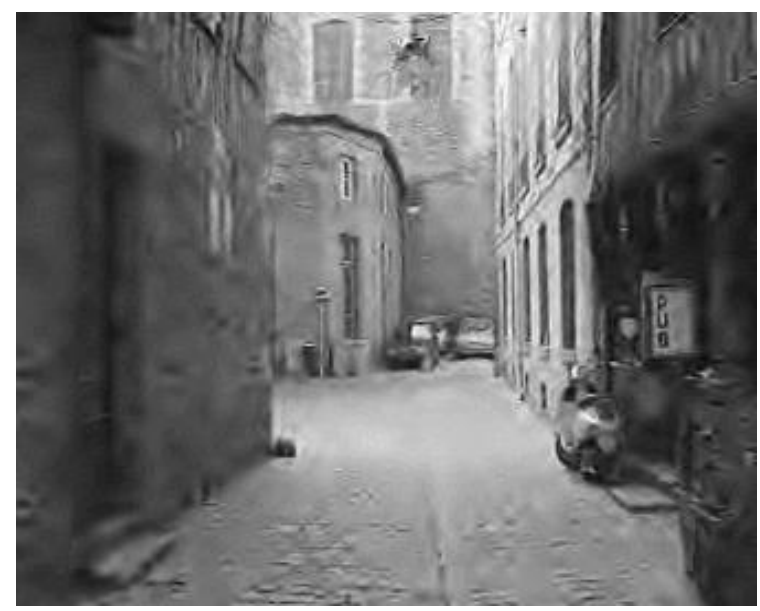

Figure 17: Street video sequence at 16kb/s: image 100 reconstructed by Rec3D coder.
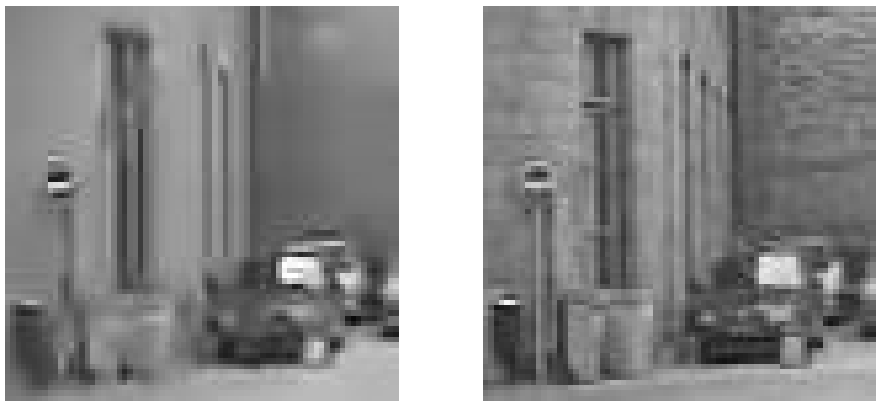

Figure 18: Street video sequence at $82 \mathrm{~kb} / \mathrm{s}$ : zoom on image 100 reconstructed by $\mathrm{H} 26 \mathrm{~L}$ (left) and Rec3D (right) coder.

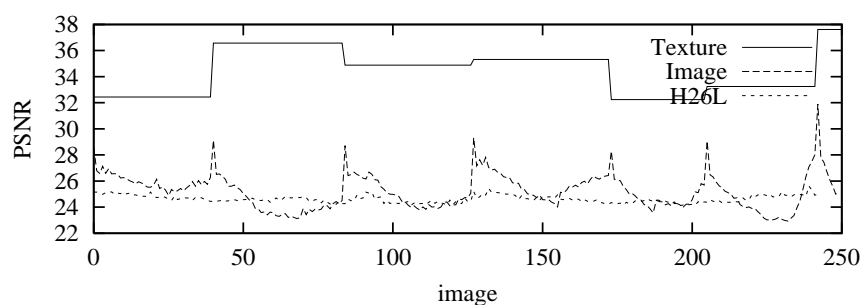

Figure 19: PSNR of Street video sequence at $82 \mathrm{~kb} / \mathrm{s}$ : comparison between H26L and Rec3d method.

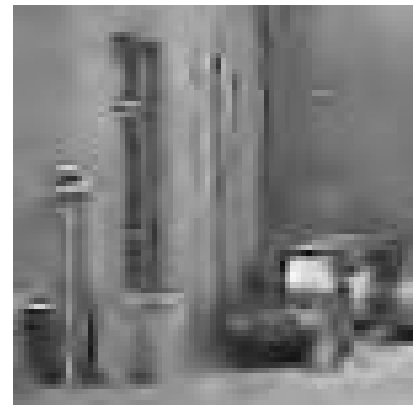

Figure 20: Street video sequence at 16kb/s: zoom on image 100 reconstructed by Rec3D coder.

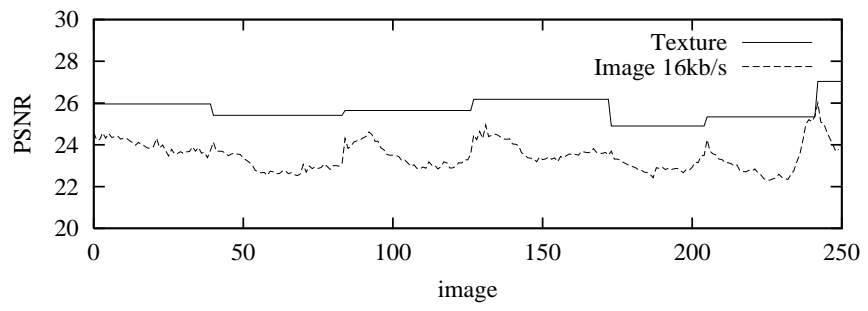

Figure 21: PSNR of Street video sequence at $16 \mathrm{~kb} / \mathrm{s} \mathrm{com-}$ pressed with Rec3D method. 